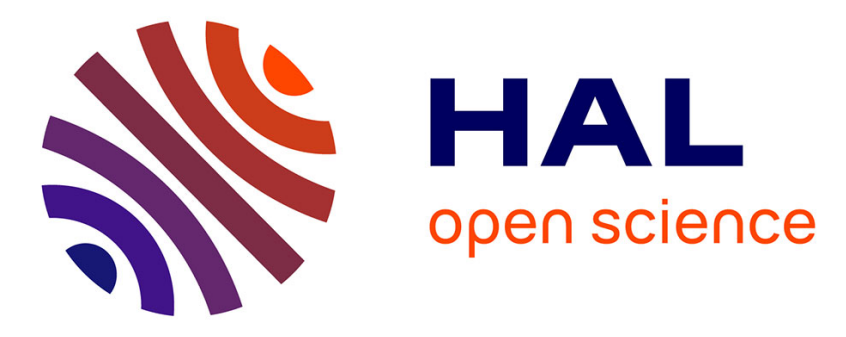

\title{
Experimental and Theoretical Infrared Signatures of REMO3 ( RE = La, Pr, Nd, Sm, and M = Co, Fe) Perovskites
}

Émile Haye, Erwan André, Fabien Capon, Silvère Barrat, Marco de La Pierre, Roberto Dovesi, Cédric Carteret

\section{To cite this version:}

Émile Haye, Erwan André, Fabien Capon, Silvère Barrat, Marco de La Pierre, et al.. Experimental and Theoretical Infrared Signatures of REMO3 ( $\mathrm{RE}=\mathrm{La}, \mathrm{Pr}, \mathrm{Nd}, \mathrm{Sm}$, and M = Co, Fe) Perovskites. Journal of Physical Chemistry C, 2018, 122 (19), pp.10519 - 10525. 10.1021/acs.jpcc.8b01433 . hal01795854

\section{HAL Id: hal-01795854 \\ https://hal.univ-lorraine.fr/hal-01795854}

Submitted on 13 Jun 2019

HAL is a multi-disciplinary open access archive for the deposit and dissemination of scientific research documents, whether they are published or not. The documents may come from teaching and research institutions in France or abroad, or from public or private research centers.
L'archive ouverte pluridisciplinaire HAL, est destinée au dépôt et à la diffusion de documents scientifiques de niveau recherche, publiés ou non, émanant des établissements d'enseignement et de recherche français ou étrangers, des laboratoires publics ou privés. 


\section{Experimental and Theoretical Infrared Signatures of}

\section{$\mathrm{REMO}_{3}(\mathrm{RE}=\mathrm{La}, \mathrm{Pr}, \mathrm{Nd}, \mathrm{Sm}$ and $\mathrm{M}=\mathrm{Co}, \mathrm{Fe})$}

\section{Perovskites}

Emile HAYE ${ }^{l}$, Erwan ANDRE* ${ }^{2}$, Fabien CAPON $^{l}$, Silvère BARRAT ${ }^{l}$, Marco DE LA PIERRE ${ }^{3}$, Roberto DOVESI ${ }^{4}$ Cédric CARTERET ${ }^{2}$

${ }^{1}$ Institut Jean Lamour, UMR 7198, CNRS-Université de Lorraine, Parc de Saurupt 54011 Nancy Cedex, FRANCE.

${ }^{2}$ Laboratoire de Chimie Physique et Microbiologie pour les Matériaux et l'Environnement, UMR7564, 405 Rue de Vandoeuvre, 54601 Villers-lès-Nancy, FRANCE.

${ }^{3}$ Curtin Institute for Computation, School of Molecular and Life Sciences, Curtin University, P.O. Box U1987, Perth, WA 6845, AUSTRALIA.

${ }^{4}$ Dipartimento di Chimica, Università di Torino and NIS (Nanostructured Interfaces and Surfaces) Center of Excellence, Via P. Giuria 7, 10125 Torino, ITALY.

\section{Abstract}

The aim of this work is to investigate the consequences of lattice distortion on the vibrational features of rare earth perovskites. To this end, a series of $\mathrm{REMO}_{3}$ compounds has been synthesized with different rare earths $(\mathrm{RE}=\mathrm{La}, \mathrm{Pr}, \mathrm{Nd}, \mathrm{Sm})$ and different transition metals 
$(\mathrm{M}=\mathrm{Fe}, \mathrm{Co})$ in order to evaluate their respective role on lattice distortion. Thin films of these materials have been deposited by magnetron co-sputtering in reactive mode and followed by an annealing in air with the same experimental conditions. Characterizations including EDX spectroscopy, X-Ray diffraction and FTIR have been completed by DFT periodic calculations in order to investigate the octahedra tilt angles and to assign the vibrational spectra. The decrease of $\mathrm{RE}$ radius enhances the distortion of the chains of octahedra that can be followed both through the calculated mean tilt angle and experimentally by the profile of the M-O-M bending region. The magnitude of the distortion depends on the dimension of the cuboctahedric site hosting the $\mathrm{RE}$ that is controlled by the $\mathrm{M}$ radius.

\section{Introduction}

The $\mathrm{ABX}_{3}$ perovskite structure is the subject of a huge number of studies firstly on inorganic perovskites, ${ }^{1-4}$ and more recently on organic perovskites ${ }^{5-7}$. The fascination with this structure is explained by its incredible capacity to accommodate elements of different nature that allows them to be used in numerous smart applications, as piezoelectric, dielectric, magnetic, catalytic or thermochromic materials.

Among the perovskites family, rare-earth cobaltites and orthoferrites $\left(\mathrm{RECoO}_{3}\right.$ and $\mathrm{REFeO}_{3}$ with $\mathrm{RE}=$ Rare Earth) have recently raised a large interest in the scientific community for their application in water splitting and photocatalytic activity ${ }^{8-12}$. Their structure and their electronic properties can be tuned depending on the size of A-cations, and the electronic state of the transition metal B, that will influence the bandgap and the photocatalytic properties. In addition, they present a good stability, which make them promising candidate for future production of hydrogen. By playing with the nature of A and B cations, it is possible to tune these properties. 
The crystal structure of $\mathrm{REMO}_{3}$ systems (with $\mathrm{RE}=\mathrm{La}, \mathrm{Pr}, \mathrm{Nd}, \mathrm{Sm}$ and $\mathrm{M}=\mathrm{Fe}, \mathrm{Co}$ ) presents a distortion with respect to an ideal cubic perovskite. Almost all of these compounds crystallize in an orthorhombic lattice (space group Pnma), except for $\mathrm{LaCoO}_{3}$, which presents a rhombohedral lattice (space group $\mathrm{R} \overline{3} \mathrm{c}$ ). All these systems can be seen as a stacking of $\mathrm{MO}_{6}$ octahedra surrounded by RE atoms in cuboctahedric sites (see Figure 1). As the size of RE is too small to fill the site, octahedra stacking is affected. The resulting lattice distortion is correlated with a decrease of the M-O-M mean angle $\alpha$, which drives interatomic interactions of oxygen $2 p$ and iron or cobalt $3 \mathrm{~d}$ electrons responsible of the electro-optical properties of these materials.

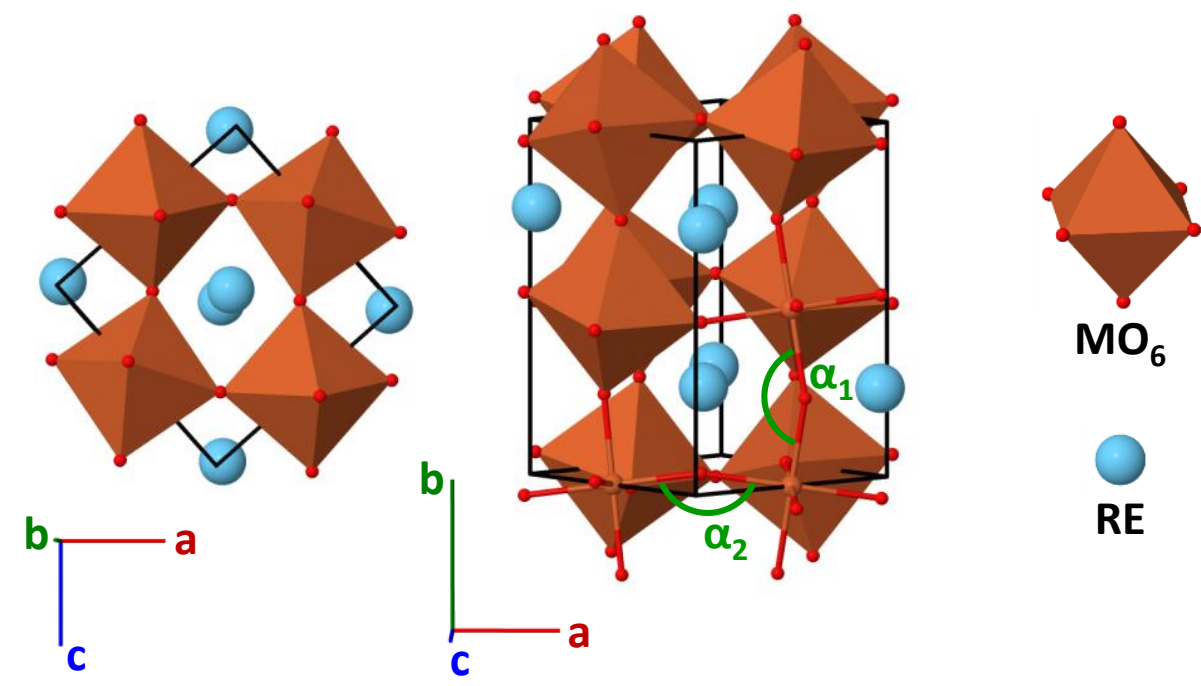

Figure 1. Representation of the orthorhombic structure of $\mathrm{REMO}_{3} . \alpha_{1}$ and $\alpha_{2}$ are the distortion angles of the chains of octahedra.

In order to link the chemical properties of these materials to the perovskite structure, XRD measurements are widely used. However, such method presents a limited use on nanomaterials or thin films, which are their most common forms for energy applications. Infrared spectroscopy 
(IR) is a simple and widespread method that can be used to probe the structural features of a system at different scales, by studying its vibrational signatures.

The vibrational properties (experimental and theoretical) of $\mathrm{REFeO}_{3}$ and $\mathrm{RECoO}_{3}$ systems have been discussed over the years ${ }^{13-20}$, but most of the work found in the literature focuses only on one or two specific compounds. Only two studies explore the effect of lattice distortion brought by the $\mathrm{RE}^{3+}$ cation on the vibrational features of these systems: Sudheendra et al. ${ }^{21}$ followed the thermal evolution of the infrared signatures of $\mathrm{RECoO}_{3}(\mathrm{RE}=\mathrm{La}, \mathrm{Pr}, \mathrm{Nd})$ systems, while Weber et $a .^{22}$ focused on the attribution of the Raman signatures of $\mathrm{REFeO}_{3}(\mathrm{RE}=\mathrm{La}$, $\mathrm{Sm}, \mathrm{Eu}, \mathrm{Gd}, \mathrm{Tb}, \mathrm{Dy})$ systems. However both of these studies focus only on the role of the $\mathrm{A}^{3+}$ cation and none of them considered that of the $\mathrm{B}^{3+}$ cation.

A previous work of some of the present authors ${ }^{23,24}$ has brought out the evolution of electrical and optical properties in the $\mathrm{RECoO}_{3}(\mathrm{RE}=\mathrm{La}, \mathrm{Nd}, \mathrm{Sm})$ and $\mathrm{REFeO}_{3}(\mathrm{RE}=\mathrm{La}, \mathrm{Pr}, \mathrm{Nd}, \mathrm{Sm})$ series, respectively. Although the modification of the Infrared features has been highlighted in these series, the complexity of the spectra (broad bands with multiple contributions) prevented a full exploitation of the vibrational information.

In the present work, we aim to investigate by Infrared spectroscopy the influence of lattice deformation brought by substitution in both $\mathrm{A}$ and $\mathrm{B}$ sites of $\mathrm{REMO}_{3}$ perovskite systems $(\mathrm{RE}=$ $\mathrm{La}, \mathrm{Pr}, \mathrm{Nd}, \mathrm{Sm}$ and $\mathrm{M}=\mathrm{Co}, \mathrm{Fe})$. The experimental data will be complemented by the simulation of theoretical spectra, calculated in the framework of density functional theory (DFT). This joint approach will make it possible to put in relation the structural and the vibrational modifications.

\section{Experimental details}

$\mathrm{REMO}_{3}(\mathrm{RE}=\mathrm{La}, \mathrm{Pr}, \mathrm{Nd}, \mathrm{Sm}$ and $\mathrm{M}=\mathrm{Fe}, \mathrm{Co})$ thin films were synthesized in a $40 \mathrm{dm}^{3}$ sputtering chamber in base vacuum of about $10^{-5} \mathrm{~Pa}$, achieved by primary and turbomolecular 
pumps. Films were deposited by magnetron co-sputtering, from pure metallic target (GoodFellow ${ }^{\circledR} 99.95 \%$ purity, diameter of $5 \mathrm{~cm}$ ), in reactive atmosphere with an $\mathrm{Ar} / \mathrm{O}_{2}$ flow rate ratio of 21/7 sccm (standard cubic centimeter per minutes), on (100) undoped silicon substrates, at a working pressure of $0.5 \mathrm{~Pa}$. The parameters for the discharge have been fixed at a current of $0.2 \mathrm{~A}$ on Fe target, and a power of $51 \mathrm{~W}$ on Co target. Concerning RE a discharged power of 110 $\mathrm{W}$ has been applied on $\mathrm{La}$, and $70 \mathrm{~W}$ on $\mathrm{Pr}, \mathrm{Nd}$ and $\mathrm{Sm}$ targets (summarize in Table 1). Note that, a power regulation is preferred for rare-earth elements and cobalt to reduce electrical instabilities. The target-substrate distance has been set at $7 \mathrm{~cm}$ for transition metal targets (Fe and Co), and at $5.5 \mathrm{~cm}$ for RE. The deposition time has been fixed to $5 \mathrm{~h}$ in order to reach a thickness of $1 \mu \mathrm{m}$. For $\mathrm{REFeO}_{3}$, this large thickness generates residual stress and promotes buckling delamination. To avoid this, a deposition temperature of $400^{\circ} \mathrm{C}(673 \mathrm{~K})$ has been used, thereby minimizing the residual compressive stresses generated during the annealing (due to the difference of thermal dilatation). For $\mathrm{RECoO}_{3}$, this effect is not significant, and the film has been deposited at room temperature. In both deposition temperatures $\left(400^{\circ} \mathrm{C}\right.$ or $\left.\mathrm{RT}\right)$, the thin films remain amorphous and, as mentioned above, they have been crystallized during $5 \mathrm{~h}$ at $700^{\circ} \mathrm{C}(973$ K). Different deposition temperatures, annealing times and annealing temperatures have been tested without significant effect on the vibrational features. After annealing, thin films exhibit a dense equiaxed grain morphology.

Table 1. Deposition conditions of $\mathrm{REMO}_{3}$ thin films

\begin{tabular}{|l|l|l|}
\hline Parameters & $\mathrm{REFeO}_{3}$ thin films & $\mathrm{RECoO}_{3}$ thin film \\
\hline \multirow{3}{*}{ Target discharge parameter } & $\mathrm{Fe}: 0.2 \mathrm{~A}$ & $\mathrm{Co}: 51 \mathrm{~W}$ \\
\cline { 2 - 3 } & $\begin{array}{l}\mathrm{La}: 110 \mathrm{~W} \\
\mathrm{Pr}, \mathrm{Nd}, \mathrm{Sm}: 70 \mathrm{~W}\end{array}$ \\
\hline Target distance & Metal: $7 \mathrm{~cm}$ & \\
\hline
\end{tabular}




\begin{tabular}{|l|l|l|}
\hline & RE: $5.5 \mathrm{~cm}$ \\
\hline Flow rate $\mathrm{Ar} / \mathrm{O}_{2}$ & $21 / 7 \mathrm{sccm}$ \\
\hline Deposition pressure & $0.5 \mathrm{~Pa}$ \\
\hline Deposition time & $5 \mathrm{~h}$ & \multicolumn{2}{|l|}{ Room temp. } \\
\hline Deposition temperature & $400^{\circ} \mathrm{C}$ \\
\hline Annealing & $700^{\circ} \mathrm{C}-5 \mathrm{~h}$, in air \\
\hline
\end{tabular}

The structure of the films has been analyzed by X-ray diffraction on a Bruker D8 diffractometer, with $2 / 2 \theta$ Bragg-Bentano geometry $(\mathrm{Cu} \mathrm{K} \alpha=1.54056 \AA)$, at room temperature. Due to a slight texturation of the film, a complete Rietveld refinement could not be performed. However, precise lattice parameters could be obtained from the XRD measurement. Structure refinement has been done using FullProf software, with 11 parameters, including background, zero, scale, lattice parameter and peak shape coefficients.

The infrared spectra in the range $30-700 \mathrm{~cm}^{-1}$ was recorded in transmission mode by accumulating 200 scans with a resolution of $4 \mathrm{~cm}^{-1}$ using a Nicolet 8700 Fourier transform infrared spectrometer with a Globar source, a DTGS-PE detector and a solid substrate beam splitter $^{\mathrm{TM}}$. A Si wafer was used as reference sample.

\section{Computational details}

Calculations were performed with the CRYSTAL14 periodic ab initio code, ${ }^{25,26}$ by using the hybrid PBE0 functional ${ }^{27}$ that has been shown to provide reliable results for the structural and electronic properties of perovskites systems. ${ }^{28}$ All electron basis sets have been used to describe non lanthanoids atoms with a contraction scheme of (8s)-(6411sp)-(411d)-(1f) for Fe and Co, and (8s)-(51sp)-(1d) for O. The exponents (in bohr ${ }^{-2}$ ) of the most diffuse functions are $\alpha_{\mathrm{sp}}=0.60$, $\alpha_{\mathrm{d}}=0.22, \alpha_{\mathrm{f}}=0.70$ for Co, $\alpha_{\mathrm{sp}}=0.55$ and $\alpha_{\mathrm{d}}=0.25, \alpha_{\mathrm{f}}=0.70$ for Fe and $\alpha_{\mathrm{sp}}=0.185$ and $\alpha_{\mathrm{d}}=$ 
0.50 for $\mathrm{O}$. In the case of Lanthanoids, pseudopotentials ${ }^{29-32}$ have been used to describe core and f electrons. They are combined with different basis sets that we have reoptimized for the purpose of calculation in crystals. Contraction schemes for $\mathrm{La}, \mathrm{Pr}, \mathrm{Nd}$ and $\mathrm{Sm}$ valence electron basis sets are the following: (311s)-(211p)-(21d). The exponents (in bohr ${ }^{-2}$ ) of the most diffuse functions are: $\alpha_{\mathrm{s}}=0.17, \alpha_{\mathrm{p}}=0.25$ and $\alpha_{\mathrm{d}}=0.40$ for La $; \alpha_{\mathrm{s}}=0.22, \alpha_{\mathrm{p}}=0.25$ and $\alpha_{\mathrm{d}}=0.32$ for Pr $; \alpha_{\mathrm{s}}=$ $0.29, \alpha_{p}=0.25$ and $\alpha_{d}=0.30$ for $\mathrm{Nd} ; \alpha_{s}=0.28, \alpha_{p}=0.27$ and $\alpha_{d}=0.32$ for Sm.

The level of accuracy in evaluating the Coulomb and Hartree-Fock exchange series is controlled by five parameters, ${ }^{25}$ for which the 8881020 values were used. The threshold on the SCF energy (SCF: self-consistent field) was set to $10^{-8}$ Ha for the geometry optimization and $10^{-}$ ${ }^{10} \mathrm{Ha}$ for the frequency calculation. The reciprocal space was sampled according to a sublattice with shrinking factor set to 8 (along the 3 lattice vectors), corresponding to 170 independent $\mathrm{k}$ vectors in the irreducible part of the Brillouin zone. The DFT exchange-correlation contribution is evaluated by numerical integration through a $(75,974)$ pruned grid (XLGRID keyword) over the unit cell volume.

The presence of $\mathrm{Fe}(\mathrm{III})$ and $\mathrm{Co}(\mathrm{III})$ in the studied compounds raises the question of their spin state, and at longer range, the question of spin ordering between $\mathrm{M}$ centers in those structures. For the construction of all model structures in this work, we decided to only consider the low temperature case $(0 \mathrm{~K})$. In this case, the preferred spin state of $\mathrm{Fe}\left(\mathrm{d}^{5}\right)$ is HS and the one of Co $\left(d^{6}\right)$ is LS, the calculated atomic magnetic moments obtained after optimization for the different compounds remain close to ideal values $\left(4.24 \mu_{\mathrm{B}}\right.$ for Fe and $0.00 \mu_{\mathrm{B}}$ for Co) and are not affected by lattice distortion. To model different spin ordering in $\mathrm{REFeO}_{3}$, the lattice symmetry has been reduced from Pnma (space group $n^{\circ} 62$ ) to $\mathrm{P} 2{ }_{1} / \mathrm{c}$ (space group $\left.\mathrm{n}^{\circ} 14\right)$. Different spin distributions have been considered for Fe (FM, A-AFM, C-AFM and G-AFM). For all studied compounds, 
the most stable structure is for a G-AFM ordering, which will be the only case presented in this work. One should mention though that the structural parameters remain close to those calculated in Pnma ( $<1 \%$ difference).

The calculation of vibrational frequencies at the $\Gamma$ point is performed within the harmonic approximation. Integrated intensities for IR absorption are evaluated through the Berry phase

approach. $^{33}$ Spectra are reconstructed from the calculated frequencies and intensities by superposition of pseudo-Voigt functions with a constant FWHM of $10 \mathrm{~cm}^{-1}$. To evaluate the contributions of the different atoms to the normal modes, isotopic substitutions have also been realized for $\mathrm{LaFeO}_{3}$ : The mass of Fe and $\mathrm{O}$ atoms (at each Wyckoff position i.e 4c and $8 \mathrm{~d}$ of the Pnma space group) has been raised by $20 \%$ while the one of La has been increased from 139 to $10000 \mathrm{u}$ in order to remove all coupling with other modes and thus reveal its participation in those modes. For each substitution, the IR spectrum is reconstructed from the same Hessian matrix than the former calculation.

\section{Results and discussion}

\section{Crystal structure of $\mathrm{REMO}_{3}$}

The first step of this work is to construct reliable model structures from which the infrared data will be calculated. The experimental XRD patterns are reported on Figure 2. Only peaks corresponding to perovskite are detected in all $\mathrm{REMO}_{3}$ corresponding to orthorhombic crystal structures except for $\mathrm{LaCoO}_{3}$, which crystallize in rhombohedral system. As the RE atomic radius decreases (from $\mathrm{La}$ to $\mathrm{Sm}$ ), peaks splitting is observed due to higher distortion of the system. This effect is more visible on ferrite system, where 21 diffraction peaks are identified in $\mathrm{SmFeO}_{3}$, while only 12 peaks are detected for $\mathrm{LaFeO}_{3}$ film. 

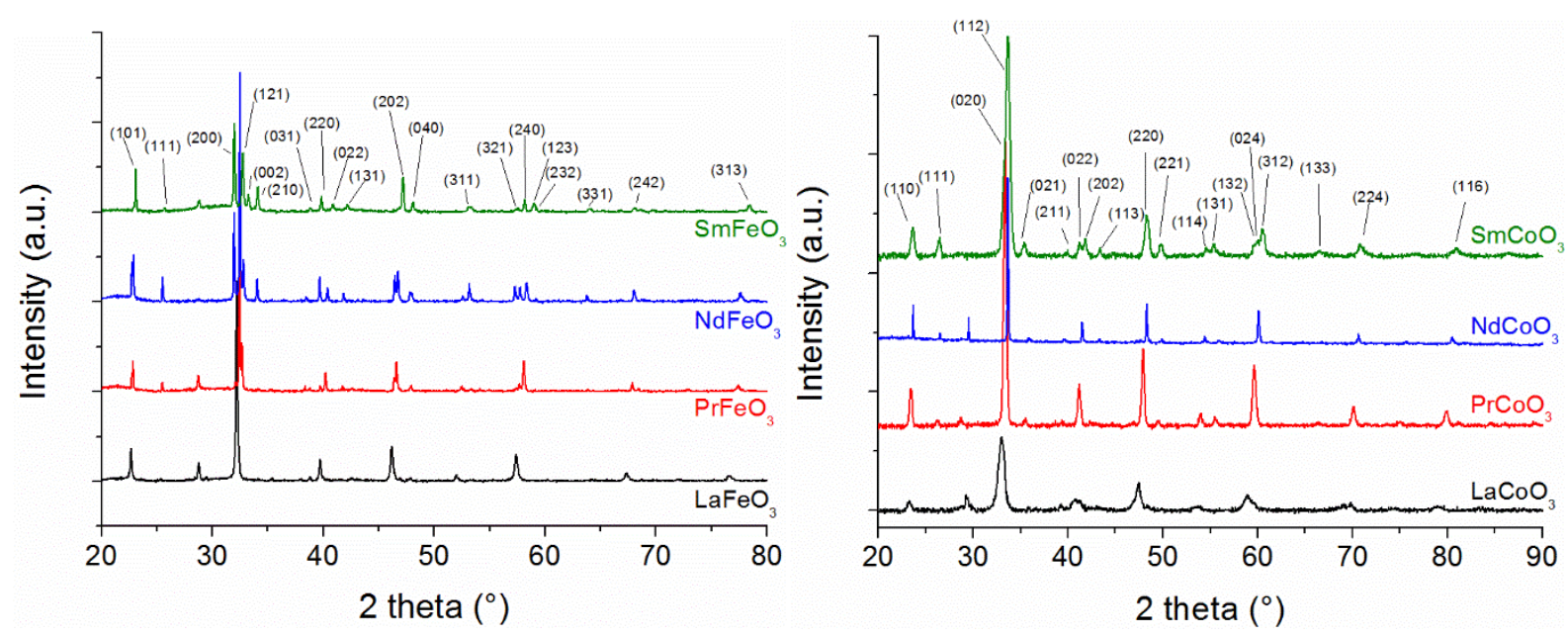

Figure 2. Experimental $\mathrm{XRD}$ patterns of $\mathrm{REFeO}_{3}$ and $\mathrm{RECoO}_{3}$ thin films

As mentioned previously, a complete Rietveld refinement with atomic positions and angles determination cannot be performed due to slight texturation of the film. Indeed, texturation influences the peak intensity, which is used to determine the atomic peak positions. However, since lattice parameters are only related to peak positions (in $2 \theta$ ), their precise determination can be performed. Experimental lattice parameters are reported in Table 2 and are compared to the optimized crystal parameters calculated in DFT. For sake of comparison, selected data obtained from the literature are also reported.

Table 2. Crystal parameters for $\mathrm{ReMO}_{3}$ perovskites in space group Pnma ( $\left.{ }^{\circ} 62\right)$ except for $\mathrm{LaCoO}_{3}\left(\mathrm{R} \overline{\mathbf{c}} \mathrm{c}, \mathrm{n}^{\circ} 167\right.$, hexagonal axes$)$. 


\begin{tabular}{|c|c|c|c|c|c|c|c|c|c|}
\hline & & $\underline{\mathrm{LaFeO}_{3}}$ & $\mathrm{PrFeO}_{3}$ & $\mathrm{NdFeO}_{3}$ & $\mathrm{SmFeO}_{3}$ & $\underline{\mathrm{LaCoO}_{3}}$ & $\mathrm{PrCoO}_{3}$ & $\mathrm{NdCoO}_{3}$ & $\mathrm{SmCoO}_{3}$ \\
\hline & Theo & 5.55 & 5.60 & 5.60 & 5.61 & 5.37 & 5.29 & 5.29 & 5.28 \\
\hline $\mathrm{a}$ & Exp & 5.57 & 5.61 & 5.60 & 5.62 & 5.38 & 5.36 & 5.33 & 5.31 \\
\hline & Ref & $5.56(i)$ & 5.57 (i) & 5.59 (i) & 5.59 (i) & $5.44(j)$ & $5.34(k)$ & $5.33(k)$ & $5.35(k)$ \\
\hline & |Theo & 7.83 & 7.79 & 7.79 & 7.73 & - & 7.53 & 7.53 & 7.50 \\
\hline$b$ & Exp & 7.86 & 7.81 & 7.82 & 7.72 & - & 7.59 & 7.55 & 7.50 \\
\hline & Ref & 7.86 (i) & $7.79(\mathrm{i})$ & $7.76(\mathrm{i})$ & $7.71(\mathrm{i})$ & - & $7.58(k)$ & $7.55(k)$ & $7.50(k)$ \\
\hline & |Theo & 5.57 & 5.51 & 5.50 & 5.44 & 13.00 & 5.38 & 5.38 & 5.32 \\
\hline$c$ & Exp & 5.57 & 5.48 & 5.51 & 5.46 & 13.31 & 5.38 & 5.34 & 5.27 \\
\hline & Ref & 5.55 (i) & $5.48(i)$ & 5.45 (i) & 5.40 (i) & $13.10(j)$ & $5.38(k)$ & $5.35(k)$ & $5.29(k)$ \\
\hline & $\mid$ Theo & 159.5 & 154.2 & 154.0 & 151.0 & 169.2 & 162.0 & 161.8 & 158.1 \\
\hline $\mathrm{d}(\mathrm{M}-\mathrm{O}) \min$ & Theo & 1.99 & 2.00 & 2.00 & 2.00 & 1.90 & 1.91 & 1.91 & 1.91 \\
\hline $\mathrm{d}(\mathrm{M}-\mathrm{O}) \max$ & Theo & 1.99 & 2.01 & 2.01 & 2.02 & 1.90 & 1.91 & 1.91 & 1.91 \\
\hline
\end{tabular}

Lattice parameters (in $\AA$ ), $\alpha$ mean distortion angle (in ${ }^{\circ}$ ), and extremum values of M-O distances d(M-O) (in $\AA$ ). (i), (j) and (k) are reported from ref ${ }^{34},{ }^{35}$ and ${ }^{36}$ respectively.

A very good agreement between theoretical and experimental results is observed (about $1 \%$ difference on average) except for $\mathrm{LaCoO}_{3}$, for which the $\mathrm{c}$ parameter is calculated shorter than what has been measured. This parameter is known to be very sensitive to the temperature ${ }^{35}$ as the spin state of Co undergo a spin transition from low to intermediate spin state (LS-IS) above 100 K. However it is not possible to model the IS state with a monodeterminental approach ${ }^{37}$. This limits our model to the LS case, corresponding to a structure at $0 \mathrm{~K}$. The data from the neutron diffraction study of Bull et al. ${ }^{35}$ reported in Table 2 are those measured at $300 \mathrm{~K}$ (to be comparable to the other compounds), but the parameters have also been measured at $4 \mathrm{~K}$ ( $\mathrm{a}=$ $5.42 \AA$ and $\mathrm{c}=12.98 \AA$ ) which are in good agreement with the calculated results.

In the Pnma space group, $\mathrm{REMO}_{3}$ structure contains two non-equivalent oxygen atoms located in Wyckoff sites 4c and 8d that lead to two different M-O-M angles. However, calculated differences between those two angles are never larger than $3^{\circ}$. Thus for the sake of clarity, only the mean values of the distortion angle $(\alpha)$ are reported in Table 2 . This angle is a good indicator 
of the lattice deformation: In a cubic system, $\alpha$ is equal to $180^{\circ}$ and as its value diminishes, the system becomes more and more distorted. As expected for both $\mathrm{RECoO}_{3}$ and $\mathrm{REFeO}_{3}$ systems, a decrease of the $\mathrm{RE}$ ionic radius (from $\mathrm{La}$ to $\mathrm{Sm}$ ) is correlated with a reduction of $\alpha$ calculated values. On the contrary, when the $\mathrm{M}$ ionic radius decreases (from Fe to $\mathrm{Co}$ ) an increase of $\alpha$ is observed. As M-O bonds get shorter (from $\sim 2 \AA$ for Fe-O to $\sim 1.9 \AA$ for $\mathrm{Co}-\mathrm{O}$ ), the size of the cuboctahedric site diminishes and it becomes more adapted to the RE. So by changing the nature of RE and $\mathrm{M}$ cations, one can span over $19^{\circ}$ of distortion, from the least distorted structure $\mathrm{LaCoO}_{3}$ to the most distorted one $\mathrm{SmFeO}_{3}$.

\section{Vibrational analysis of $\mathrm{REFeO}_{3}$}

The first results presented here are the substitution of $\mathrm{A}$ cation in $\mathrm{REFeO}_{3}$. In the conditions where experimental spectra have been recorded $(298 \mathrm{~K}, 1 \mathrm{~atm})$ all the compounds of this series are in the same spin state (HS state for Fe atoms with G-AFM ordering) and crystallize in the same space group (Pnma). Their IR spectra will thus possess the same number of active normal modes, which will ease their comparison.

In cubic $(\mathrm{Pm} \overline{3} \mathrm{~m}) \mathrm{ABO}_{3}$ perovskites there is only 3 IR active modes $\left(\Gamma=3 \mathrm{~T}_{1 \mathrm{u}}\right)$ corresponding to $\mathrm{B}-\mathrm{O}$ stretching, B-O-B bending, and the displacement of A relative to B-O lattice. If cation A is too small, the $\mathrm{BO}_{6}$ chains of octahedra are distorted, and the symmetry lowering from $\mathrm{O}_{\mathrm{h}}$ to $\mathrm{D}_{2 \mathrm{~h}}$ leads to a higher number of active modes. The selection rules for these orthorhombic perovskites in Pnma symmetry ${ }^{38}$ give the irreducible representation: $\Gamma=9 \mathrm{~B}_{1 \mathrm{u}}+7 \mathrm{~B}_{2 \mathrm{u}}+9 \mathrm{~B}_{3 \mathrm{u}}$ for a total of 25 active modes in IR (calculated wavenumbers and intensities are reported in S.I, in Table S1). In the experimental IR spectrum these vibration modes are not well separated and form bands of complex shape, as can be seen in case of $\mathrm{LaFeO}_{3}$ reported in Figure 3. In order to assign this spectrum, simulated spectra corresponding to cubic and orthorhombic structures are also reported. To complete this figure, isotopic substitutions have been realized for the different 
atoms of the Pnma cell (in which $\mathrm{La}, \mathrm{Fe}, \mathrm{O} 1$ and $\mathrm{O} 2$ occupy the Wyckoff sites 4c, 4b, 4c and 8d respectively). The bands influenced by the isotopic substitution have been assigned accordingly (the simulated spectra corresponding to these isotopic substitutions can be found in S.I. in Figure S1). The general agreement between the experimental and the theoretical spectra is good. For this reason the description of the vibrational features (and in particular the mentioned wavenumbers) will focus mainly on the experimental data, while the theoretical data will be used to refine the attribution.

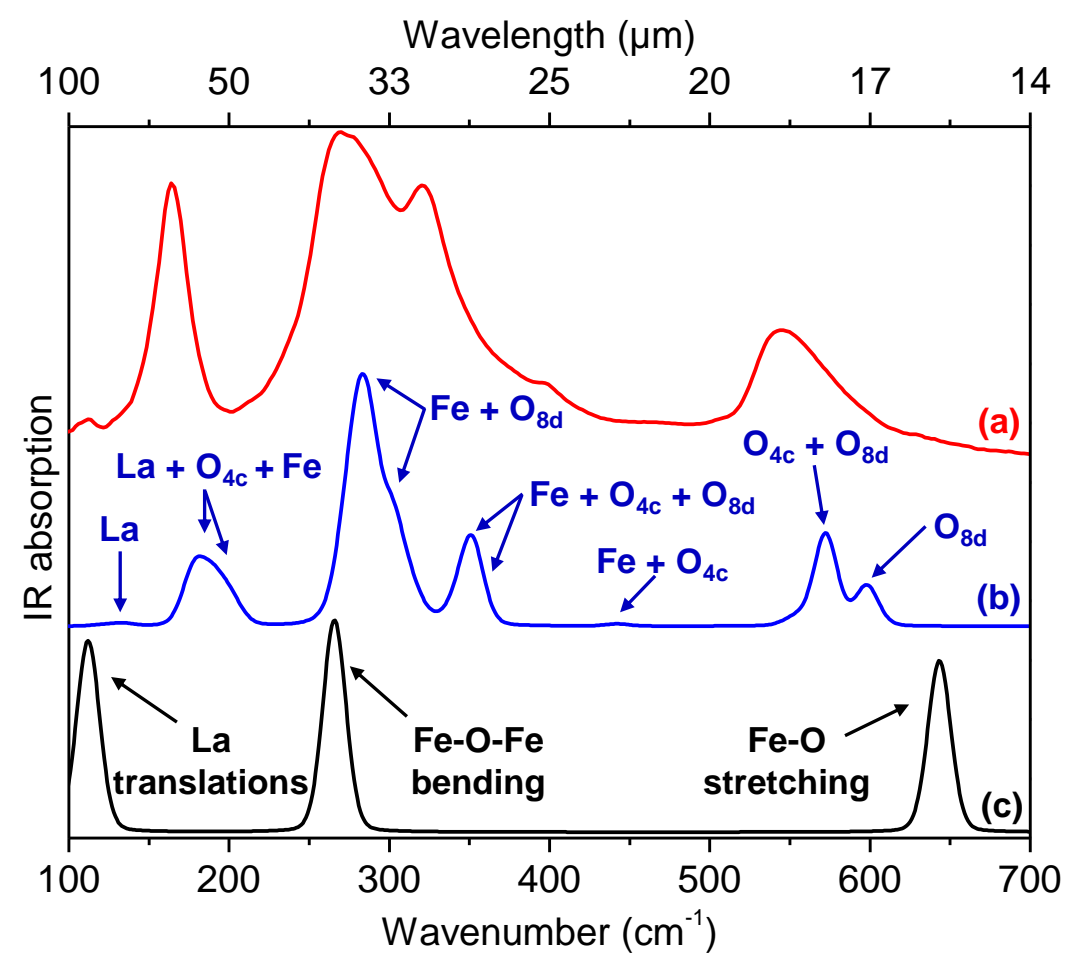

Figure 3. Infrared spectra of $\mathrm{LaFeO}_{3}$. Comparison between experimental (a) spectra and those calculated for orthorhombic (b) and cubic (c) structures. Modes are labelled according to their type and to the main atom involved.

The three groups of vibration modes are affected quite differently by the lattice deformation. The Fe-O stretching group is redshifted. It is split into two main contributions (although only one 
band is resolved on the experimental spectrum), depending on the nature of the Fe-O elongation considered: in the $(\mathrm{a}, \mathrm{c})$ plane (main contribution of $\mathrm{O}_{8 \mathrm{~d}}$ ) for the highest wavenumbers $\left(\bar{v}>550 \mathrm{~cm}^{-1}\right)$ and along $\mathrm{b}$ direction (main contribution of $\mathrm{O}_{4 \mathrm{c}}$ ) for the lowest wavenumbers $\left(\bar{v}<550 \mathrm{~cm}^{-1}\right)$. No coupling with $\mathrm{La}$ or Fe atoms is observed for these modes, we can then consider them as almost "pure modes". The group of La "translations" is more affected by the deformation with a splitting $\left(\Delta \bar{v} \sim 52 \mathrm{~cm}^{-1}\right)$ between in phase or antiphase La - La motions, with the band corresponding to in phase modes $\left(\bar{v}=164 \mathrm{~cm}^{-1}\right)$ being the most intense. Finally, the group of modes that is most affected by the lattice deformation is the group corresponding to combinations of Fe-O-Fe bending modes that split into a band of complex shape, spread over $200 \mathrm{~cm}^{-1}$. The 3 resolved bands observed in the experimental spectrum correspond to 14 vibrational modes, grouped in 7 bands in the theoretical spectrum. The most intense signal (measured at $270 \mathrm{~cm}^{-1}$ ) is similar to the $\mathrm{T}_{1 \mathrm{u}}$ bending mode of a cubic perovskite; it corresponds to vibration modes where Fe-O-Fe deformations of the different octahedra are all in phase. These deformations can be considered as "internal mode". On the contrary in the bands located at higher wavenumbers $\left(322 \mathrm{~cm}^{-1}\right.$ to $\left.400 \mathrm{~cm}^{-1}\right)$, the deformations of the different octahedra are not in phase. These modes can be considered as sort of "external mode" and be described as deformations of the chains of octahedra.

In order to follow the modifications of the vibrational features of this system as the lattice deformation increases, experimental and theoretical IR spectra of the $\mathrm{REFeO}_{3}$ series are displayed in Figure 4a and 4c respectively. Theoretical spectra reproduce correctly the evolutions of experimental spectra along the $\mathrm{REFeO}_{3}$ series, allowing the characterization of the spectral modifications. As mentioned in the previous section, numerical values correspond to the experimental data (unless specified otherwise). In Figure 4a, almost no modification is observed 
for the Fe-O stretching modes $\left(500-600 \mathrm{~cm}^{-1}\right)$. A small shift toward the low wavenumbers $\left(\Delta \bar{v} \sim 10 \mathrm{~cm}^{-1}\right)$ is observed as well as a decrease in intensity of the $\mathrm{Fe}-\mathrm{O}_{8 \mathrm{~d}}$ stretching modes.

Focusing now on the group of RE translations, one can observe that in phase translation modes $\left(150-200 \mathrm{~cm}^{-1}\right)$ are slightly blueshifted $\left(\Delta \bar{\nu} \sim 8 \mathrm{~cm}^{-1}\right)$. For these modes, two competing effects are in play as RE evolves from La to $\mathrm{Sm}$ : (i) The increase of RE mass should induce a redshift, while (ii) the decrease of the cation's radius give it more freedom of movement in the cuboctahedric site that should induce a blueshift. Hence the trend observed in the experimental spectra reveals that the second effect (ii) is dominant here. This is not the case for the calculated spectra where a slight redshift $\left(\Delta \bar{v} \sim 8 \mathrm{~cm}^{-1}\right)$ is predicted. This highlights the tendency, in the computational model, to underestimate the interactions between RE and its surrounding cage. As a final comment for this region, a shoulder is observed at $\bar{v} \sim 155 \mathrm{~cm}^{-1}$ in the $\mathrm{SmFeO}_{3}$ spectrum. This band corresponds to an out-of-phase translation mode of $\mathrm{FeO}_{6}$ octahedra planes collinear to (a,c) directions. Its position is not influenced by the nature of $\mathrm{RE}$, but in $\mathrm{LaFeO}_{3}$ it overlaps with the band corresponding to La in phase translation modes.

The spectral region corresponding to Fe-O-Fe bending modes $\left(220-420 \mathrm{~cm}^{-1}\right)$ is, as expected, the most sensitive to lattice deformations. As they increase along the series from La to Sm, the intense band corresponding to "internal" deformation modes is split in two contributions. Modes corresponding to motion in the (a,c) plane shift from $270 \mathrm{~cm}^{-1}$ in $\mathrm{LaFeO}_{3}$ to $240 \mathrm{~cm}^{-1}$ in $\mathrm{SmFeO}_{3}$, while the mode corresponding to Fe translation in the b direction remains essentially at the same position (slight blueshift $\Delta \bar{v} \sim 5 \mathrm{~cm}^{-1}$ ). But the most visible effect of the lattice deformation is observed for the "external" deformation modes of the octahedra strings $\left(300-420 \mathrm{~cm}^{-1}\right)$. All these modes are up shifted $\left(\Delta \bar{v} \sim 15 \mathrm{~cm}^{-1}\right.$ from $\mathrm{LaFeO}_{3}$ to $\left.\mathrm{SmFeO}_{3}\right)$ and they become more and 
more resolved in the experimental spectra, until every band present in the corresponding calculated spectrum is resolved.
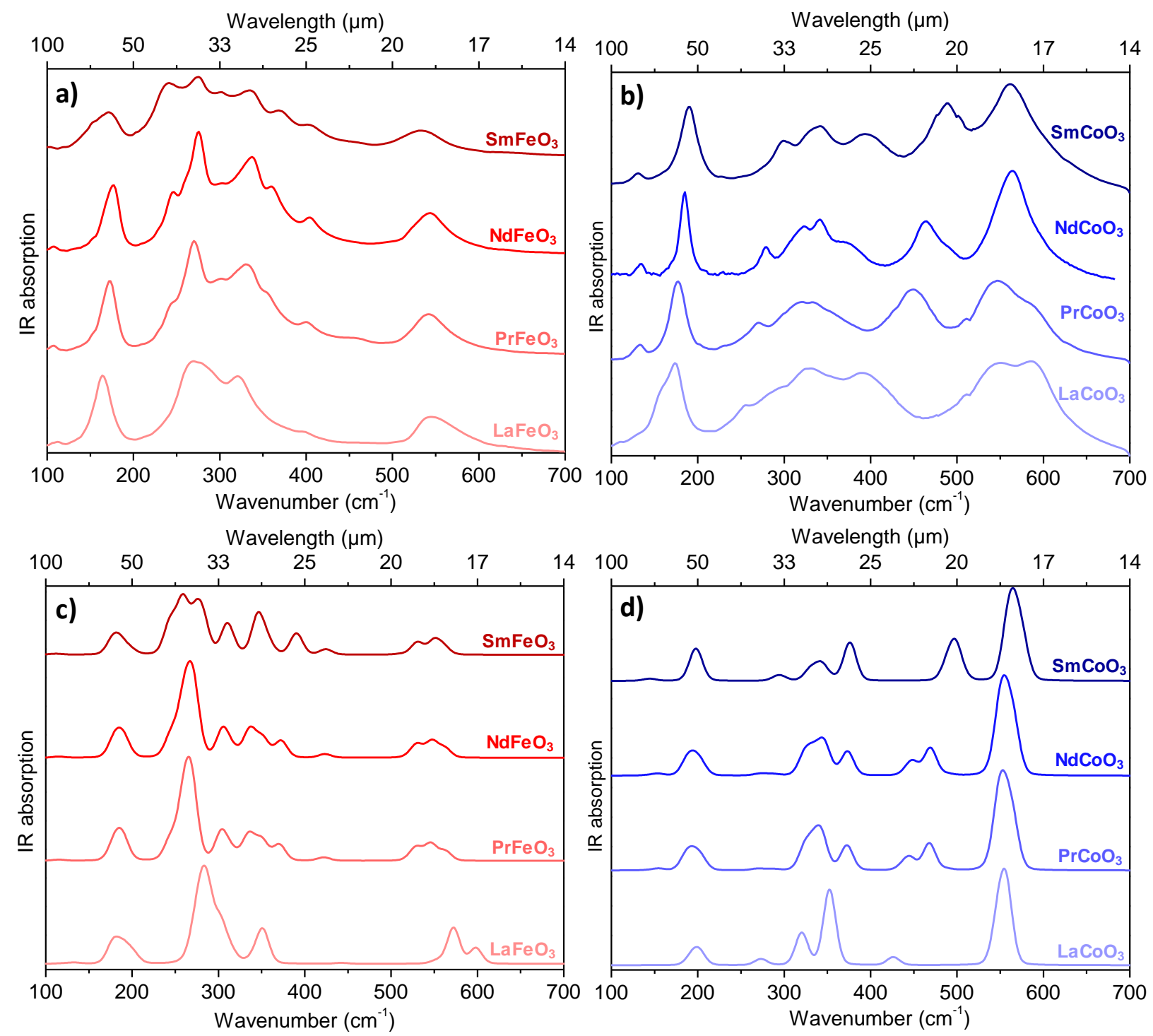

Figure 4. Infrared spectra of $\mathrm{REMO}_{3}$. Comparison between experimental $(\mathrm{a}, \mathrm{b})$ and simulated (c,d) data.

Fe or Co in B site

$\mathrm{SmFeO}_{3}$ is the most distorted system considered in this study. It can be used as a reference compound to study the influence of $\mathrm{B}$ cation on $\mathrm{REMO}_{3}$ vibrational signature. Changing the 
transition-metal Fe by $\mathrm{Co}$ in $\mathrm{SmMO}_{3}$, the lattice distortion will decrease without changing the system symmetry or the number of active modes (and their nature). The shifts calculated for these different modes when passing from $\mathrm{SmFeO}_{3}$ to $\mathrm{SmCoO}_{3}$ are shown in Figure 5 .

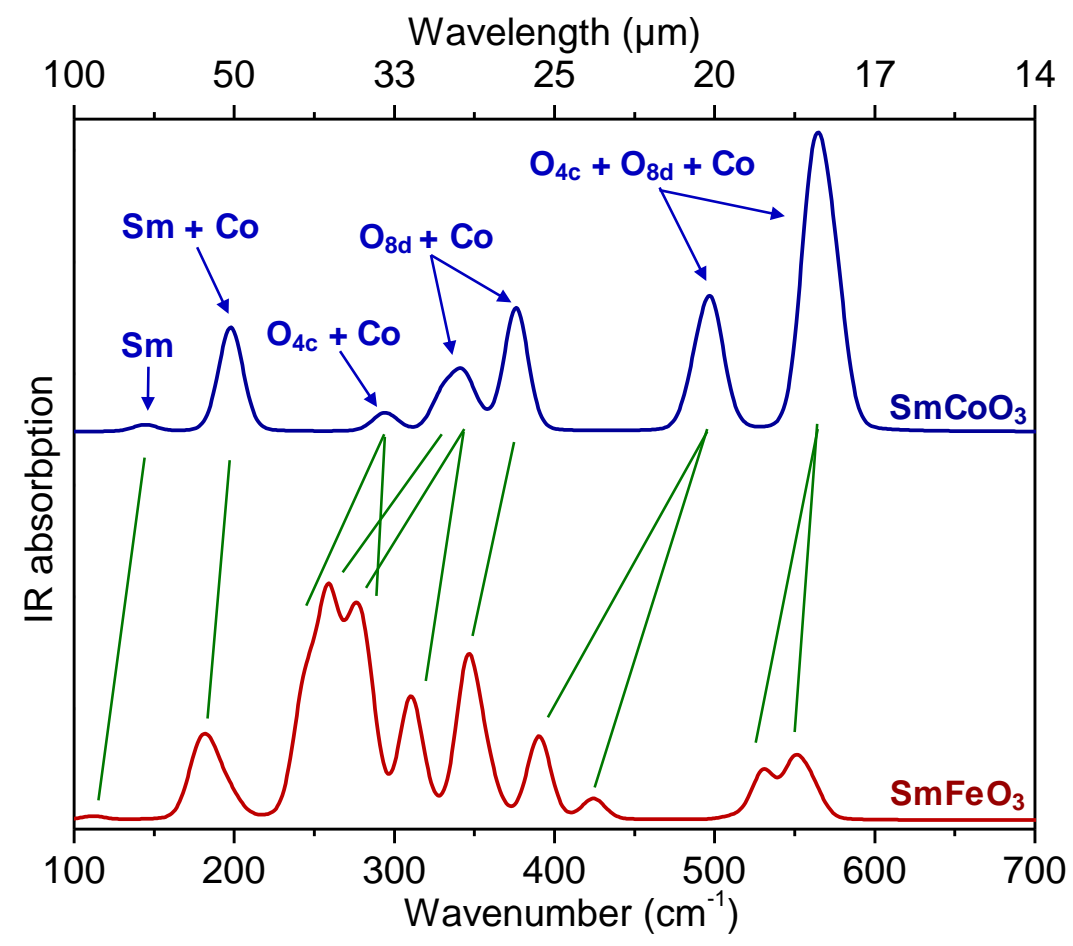

Figure 5. Relations between modes calculated of $\mathrm{SmFeO}_{3}$ and $\mathrm{SmCoO}_{3}$.

Only small modifications are observed in the M-O stretching region $\left(450-600 \mathrm{~cm}^{-1}\right)$. Since Co-O bonds are shorter than the Fe-O ones, this band is blueshifted (by $20 \mathrm{~cm}^{-1}$ in the theoretical spectrum, and $30 \mathrm{~cm}^{-1}$ in the experimental one in Figure 4b). As the $\alpha$ deformation angle is getting closer to $180^{\circ}$ in $\mathrm{SmCoO}_{3}$, the difference between $\mathrm{O}_{8 \mathrm{~d}}$ and $\mathrm{O}_{4 \mathrm{c}}$ is reduced and the two bands calculated for $\mathrm{SmFeO}_{3}$ merge in $\mathrm{SmCoO}_{3}$ (bandwidths in the experimental spectra, Figure $4 \mathrm{~b}$, do not allow to observe this phenomenon). In the region corresponding to Sm translation modes $\left(100-200 \mathrm{~cm}^{-1}\right)$ the main modification is a blueshift of the two bands $\left(\sim 20 \mathrm{~cm}^{-1}\right.$ for both theoretical and experimental spectra) that is due to the smaller size of the cuboctahedric site and 
hence a stronger coupling with the cage of $\mathrm{MO}_{6}$ octahedra. The region corresponding to $\mathrm{M}-\mathrm{O}-\mathrm{M}$ bending modes is simplified as the $\alpha$ angle increases. Modes with a strong M contribution (almost all "internal modes") form a group of three bands located between $300 \mathrm{~cm}^{-1}$ and $400 \mathrm{~cm}^{-1}$ while modes corresponding to deformations of the chains of octahedra ("external modes") merge into a separate group that is upshifted close the stretching mode region (490 and $497 \mathrm{~cm}^{-1}$ in the theoretical and the experimental spectra respectively). The position of this last band is the most sensitive to the lattice deformation as it can also be seen in the spectra of the $\mathrm{RECoO}_{3}$ series reported Figure $4 \mathrm{~b}$ and $4 \mathrm{~d}$. The trends described for the different spectral region in the $\mathrm{REFeO}_{3}$ series are still valid for $\mathrm{RECoO}_{3}$ whose spectrum is even more sensitive to RE substitution. In particular, the band corresponding to chains deformation in the experimental spectra is redshifted over $100 \mathrm{~cm}^{-1}$ between $\mathrm{SmCoO}_{3}$ and $\mathrm{LaCoO}_{3}$, compared to only $10-15 \mathrm{~cm}^{-1}$ along the iron series. However, the interpretation of $\mathrm{RECoO}_{3}$ vibrational spectra is complicated by the presence of a spin transition, for some of these compounds, near the temperature of analysis ( $298 \mathrm{~K}$ ). Indeed, it is known that $\mathrm{LaCoO}_{3}, \mathrm{PrCoO}_{3}$ and $\mathrm{NdCoO}_{3}$ undergo a LS-HS (or IS for $\mathrm{LaCoO}_{3}$ ) spin transition around $100 \mathrm{~K}, 200 \mathrm{~K}$ and $300 \mathrm{~K}$ respectively. ${ }^{21,35,39}$ This means that if the experimental IR spectrum of $\mathrm{NdCoO}_{3}$ can be considered as purely the one of $\mathrm{LS}$, those of $\mathrm{PrCoO}_{3}$ and $\mathrm{LaCoO}_{3}$ are a mix of contributions coming from different spin states. In particular, the splitting of the Co$\mathrm{O}$ stretching region in $\mathrm{LaCoO}_{3}$ has been explained by Sudheendra et al. ${ }^{21}$ as a sum of contributions coming from LS and IS states. From these results, one can assign the shoulder observed (at $\sim 600 \mathrm{~cm}^{-1}$ ) in the stretching region of $\mathrm{PrCoO}_{3}$ and $\mathrm{LaCoO}_{3}$, to modes belonging to higher spin states. This assignment is consistent with the fact that its relative intensity, with respect to the other stretching modes, is higher in $\mathrm{LaCoO}_{3}$ than in $\mathrm{PrCoO}_{3}$. And finally, this band is not present in the calculated spectra, for which only the fundamental spin state has been 
considered. The position and the trend of the band corresponding to LS state (measured at 560 $\mathrm{cm}^{-1}$ in $\mathrm{SmCoO}_{3}$ and down shifting to $547 \mathrm{~cm}^{-1}$ in $\mathrm{LaCoO}_{3}$ ) are correctly modeled.

\section{Conclusion}

In this work, thin films of $\mathrm{REMO}_{3}$ perovskites $(\mathrm{RE}=\mathrm{La}, \mathrm{Pr}, \mathrm{Nd}, \mathrm{Sm} ; \mathrm{M}=\mathrm{Co}, \mathrm{Fe}$ ) have been synthesized and characterized with the same experimental conditions. The crystallographic parameters obtained from XRD refinement for the series are in good agreement with data present in the literature and with data obtained by DFT calculations. The computational models of $\mathrm{REMO}_{3}$ series give access to other indicators of the lattice deformation such as $\alpha$, the mean angle of chains distortion. It has been shown that this angle decreases along with the RE radius (from $\mathrm{LaMO}_{3}$ to $\mathrm{SmMO}_{3}$ ) and highlights an increase of the lattice distortion. However when the $\mathrm{M}$ radius decreases (from $\mathrm{REFeO}_{3}$ to $\mathrm{RECoO}_{3}$ ), the $\alpha$ angle increases and reveals a decrease of the size of the cuboctahedric site. These structural modifications appear clearly on the vibrational features of $\mathrm{REMO}_{3}$ and their assignment has been done by relying on comparison with simulated spectra obtained from DFT periodic calculations. Lattice deformations due to the decrease of RE size are characterized by a modification of bands corresponding to RE translation modes and bands corresponding to $\mathrm{M}-\mathrm{O}-\mathrm{M}$ bending modes, both due to the bending of the $\mathrm{MO}_{6}$ chains of octahedra. Symmetry lowering of RE environment creates a splitting of out-of-phase and in phase translations modes, which is more important than the redshift due to the mass increase of RE. The splitting of the M-O-M bending region is controlled by interactions between the chains of octahedra, in phase modes are redshifted while out-of-phase modes are blueshifted. By adjusting the dimensions of the cage of octahedra around $\mathrm{RE}$, substitution of $\mathrm{Fe}^{3+}$ by $\mathrm{Co}^{3+}$ cations decreased the splitting of the perturbed regions, but amplified the influence of RE substitution. The modes that are the most sensitive to RE substitution in $\mathrm{RECoO}_{3}$ systems are 
antiphase bending of the chains of octahedra. Finally, comparison between experimental and theoretical data made it possible in $\mathrm{LaCoO}_{3}$ and $\mathrm{PrCoO}_{3}$ to assign bands corresponding to excited spin states.

\section{ASSOCIATED CONTENT}

Supporting Information. A file named Supporting_Informations.pdf is provided. It contains the vibrational information (wavenumber, intensity, symmetry) calculated for the different $\mathrm{REMO}_{3}$ systems, as well as a figure picturing the effect of isotopic substitutions on $\mathrm{LaFeO}_{3}$ calculated IR spectrum.

\section{AUTHOR INFORMATION}

\section{Corresponding Author}

*erwan.andre@univ-lorraine.fr

\section{Author Contributions}

The manuscript was written through contributions of all authors. All authors have given approval to the final version of the manuscript.

\section{ACKNOWLEDGMENT}

\section{REFERENCES}

(1) Zener, C. Interaction between the d -Shells in the Transition Metals. II. Ferromagnetic Compounds of Manganese with Perovskite Structure. Phys. Rev. 1951, 82, 403-405.

(2) Kanamori, J. Superexchange Interaction and Symmetry Properties of Electron Orbitals. $J$. Phys. Chem. Solids 1959, 10, 87-98. 
(3) Cohen, R. E. Origin of Ferroelectricity in Perovskite Oxides. Nature 1992, 358, 136-138.

(4) Peña, M. A.; Fierro, J. L. G. Chemical Structures and Performance of Perovskite Oxides. Chem. Rev. 2001, 101, 1981-2018.

(5) Kojima, A.; Teshima, K.; Shirai, Y.; Miyasaka, T. Organometal Halide Perovskites as Visible-Light Sensitizers for Photovoltaic Cells. J. Am. Chem. Soc. 2009, 131, 6050-6051.

(6) Lee, M. M.; Teuscher, J.; Miyasaka, T.; Murakami, T. N.; Snaith, H. J. Efficient Hybrid Solar Cells Based on Meso-Superstructured Organometal Halide Perovskites. Science 2012, 338, $643-647$.

(7) Xing, G.; Mathews, N.; Sun, S.; Lim, S. S.; Lam, Y. M.; Gratzel, M.; Mhaisalkar, S.; Sum, T. C. Long-Range Balanced Electron- and Hole-Transport Lengths in Organic-Inorganic CH3NH3PbI3. Science 2013, 342, 344-347.

(8) Tijare, S. N.; Joshi, M. V.; Padole, P. S.; Mangrulkar, P. A.; Rayalu, S. S.; Labhsetwar, N. K. Photocatalytic Hydrogen Generation through Water Splitting on Nano-Crystalline $\mathrm{LaFeO}_{3}$ Perovskite. Int. J. Hydrog. Energy 2012, 37, 10451-10456.

(9) Tijare, S. N.; Bakardjieva, S.; Subrt, J.; Joshi, M. V.; Rayalu, S. S.; Hishita, S.; Labhsetwar, N. Synthesis and Visible Light Photocatalytic Activity of Nanocrystalline $\mathrm{PrFeO}_{3}$ Perovskite for Hydrogen Generation in Ethanol-Water System. J. Chem. Sci. 2014, 126, $517-$ 525.

(10) Meziani, D.; Reziga, A.; Rekhila, G.; Bellal, B.; Trari, M. Hydrogen Evolution under Visible Light over $\mathrm{LaCoO}_{3}$ Prepared by Chemical Route. Energy Convers. Manag. 2014, 82, 244-249. 
(11) Yu, Q.; Meng, X.; Wang, T.; Li, P.; Liu, L.; Chang, K.; Liu, G.; Ye, J. A Highly Durable $\mathrm{P}-\mathrm{LaFeO}_{3} / \mathrm{n}-\mathrm{Fe}_{2} \mathrm{O}_{3}$ Photocell for Effective Water Splitting under Visible Light. Chem Commun 2015, 51, 3630-3633.

(12) Emery, A. A.; Saal, J. E.; Kirklin, S.; Hegde, V. I.; Wolverton, C. High-Throughput Computational Screening of Perovskites for Thermochemical Water Splitting Applications. Chem. Mater. 2016, 28, 5621-5634.

(13) Romero, M.; Gómez, R. W.; Marquina, V.; Pérez-Mazariego, J. L.; Escamilla, R. Synthesis by Molten Salt Method of the $\mathrm{AFeO}_{3}$ System (A=La, Gd) and Its Structural, Vibrational and Internal Hyperfine Magnetic Field Characterization. Phys. B Condens. Matter 2014, 443, 90-94.

(14) Zhou, S.; Shi, L.; Zhao, J.; He, L.; Yang, H.; Zhang, S. Ferromagnetism in $\mathrm{LaCoO}_{3}$ Nanoparticles. Phys. Rev. B 2007, 76, 172407.

(15) Janaki, J.; Nithya, R.; Ganesamoorthy, S.; Sairam, T. N.; Ravindran, T. R.; Vinod, K.; Bharathi, A. Study of Thermally Induced Spin State Transition in $\mathrm{NdCoO}_{3}$ Single Crystal AIP Conf. Proc. 2013, 1512, 838-839.

(16) Chanda, S.; Saha, S.; Dutta, A.; Sinha, T. P. Raman Spectroscopy and Dielectric Properties of Nanoceramic NdFeO3. Mater. Res. Bull. 2013, 48, 1688-1693.

(17) Saha, S.; Chanda, S.; Dutta, A.; Sinha, T. P. Dielectric Relaxation of $\mathrm{PrFeO}_{3}$ Nanoparticles. Solid State Sci. 2016, 58, 55-63.

(18) Kozlenko, D. P.; Golosova, N. O.; Jirák, Z.; Dubrovinsky, L. S.; Savenko, B. N.; Tucker, M. G.; Le Godec, Y.; Glazkov, V. P. Temperature- and Pressure-Driven Spin-State Transitions in $\mathrm{LaCoO}_{3}$. Phys. Rev. B 2007, 75, 064422. 
(19) Coutinho, P. V.; Cunha, F.; Barrozo, P. Structural, Vibrational and Magnetic Properties of the Orthoferrites $\mathrm{LaFeO}_{3}$ and $\mathrm{YFeO}_{3}$ : A Comparative Study. Solid State Commun. 2017, 252, 59-63.

(20) Gryaznov, D.; Evarestov, R. A.; Maier, J. Hybrid Density-Functional Calculations of Phonons in $\mathrm{LaCoO}_{3}$. Phys. Rev. B 2010, 82, 224301.

(21) Sudheendra, L.; Motin Seikh, M.; Raju, A. R.; Narayana, C. An Infrared Spectroscopic Study of the Low-Spin to Intermediate-Spin State (1A1-3T1) Transition in Rare Earth Cobaltates, $\mathrm{LnCoO}_{3}(\mathrm{Ln}=\mathrm{La}$, Pr and Nd). Chem. Phys. Lett. 2001, 340, 275-281.

(22) Weber, M. C.; Guennou, M.; Zhao, H. J.; Íñiguez, J.; Vilarinho, R.; Almeida, A.; Moreira, J. A.; Kreisel, J. Raman Spectroscopy of Rare-Earth Orthoferrites $\mathrm{RFeO}_{3}$ ( $\mathrm{R}=\mathrm{La}$, Sm, Eu, Gd, Tb, Dy). Phys. Rev. B 2016, 94, 214103.

(23) Capon, F.; Boileau, A.; Carteret, C.; Martin, N.; Boulet, P.; Pierson, J. F. Cation Size Effect on the Thermochromic Properties of Rare Earth Cobaltites $R E \mathrm{CoO}_{3}(R E: \mathrm{La}, \mathrm{Nd}, \mathrm{Sm}) . J$. Appl. Phys. 2013, 114, 113510.

(24) Haye, E.; Capon, F.; Barrat, S.; Boulet, P.; Andre, E.; Carteret, C.; Bruyere, S. Properties of Rare-Earth Orthoferrites Perovskite Driven by Steric Hindrance. J. Alloys Compd. 2016, 657, $631-638$.

(25) Dovesi, R.; Orlando, R.; Erba, A.; Zicovich-Wilson, C. M.; Civalleri, B.; Casassa, S.; Maschio, L.; Ferrabone, M.; De La Pierre, M.; D’Arco, P.; et al. CRYSTAL14: A Program for the Ab Initio Investigation of Crystalline Solids. Int. J. Quantum Chem. 2014, 114, 1287-1317.

(26) Dovesi, R.; Saunders, V. R.; Roetti, C.; Orlando, R.; Zicovich-Wilson, C. M.; Pascale, F.; Civalleri, B.; Doll, K.; Harrison, N. M.; Bush, I. J.; et al. CRYSTAL14 User's Manual; Università di Torino, Torino, 2014. 
(27) Adamo, C.; Barone, V. Toward Reliable Density Functional Methods without Adjustable Parameters: The PBE0 Model. J. Chem. Phys. 1999, 110, 6158-6170.

(28) Franchini, C. Hybrid Functionals Applied to Perovskites. J. Phys. Condens. Matter 2014, $26,253202$.

(29) Dolg, M.; Stoll, H.; Savin, A.; Preuss, H. Energy-Adjusted Pseudopotentials for the Rare Earth Elements. Theor. Chim. Acta 1989, 75, 173-194.

(30) Dolg, M.; Stoll, H.; Preuss, H. A Combination of Quasirelativistic Pseudopotential and Ligand Field Calculations for Lanthanoid Compounds. Theor. Chim. Acta 1993, 85, 441-450.

(31) Yang, J.; Dolg, M. Valence Basis Sets for Lanthanide 4f-in-Core Pseudopotentials Adapted for Crystal Orbital Ab Initio Calculations. Theor. Chem. Acc. 2005, 113, 212-224.

(32) Weigand, A.; Cao, X.; Yang, J.; Dolg, M. Quasirelativistic F-in-Core Pseudopotentials and Core-Polarization Potentials for Trivalent Actinides and Lanthanides: Molecular Test for Trifluorides. Theor. Chem. Acc. 2010, 126, 117-127.

(33) Pascale, F.; Zicovich-Wilson, C. M.; López Gejo, F.; Civalleri, B.; Orlando, R.; Dovesi, R. The Calculation of the Vibrational Frequencies of Crystalline Compounds and Its Implementation in the CRYSTAL Code. J. Comput. Chem. 2004, 25, 888-897.

(34) Morales, L.; Sierra-Gallego, G.; Barrero, C. A.; Arnache, O. Relative Recoilless FFactors in $\mathrm{REFeO}_{3}$ (RE=Rare-Earth $\mathrm{La}, \mathrm{Pr}, \mathrm{Nd}$ and $\mathrm{Sm}$ ) Orthoferrites Synthesized by SelfCombustion Method. Mater. Sci. Eng. B 2016, 211, 94-100.

(35) Bull, C. L.; Knight, K. S. Low-Temperature Structural Behaviour of $\mathrm{LaCoO}_{3}-\mathrm{A}$ HighResolution Neutron Study. Solid State Sci. 2016, 57, 38-43. 
(36) Knížek, K.; Jirák, Z.; Hejtmánek, J.; Veverka, M.; Maryško, M.; Maris, G.; Palstra, T. T. M. Structural Anomalies Associated with the Electronic and Spin Transitions in $\mathrm{LnCoO}_{3}$. Eur. Phys. J. B 2005, 47, 213-220.

(37) Křápek, V.; Novák, P.; Kuneš, J.; Novoselov, D.; Korotin, D. M.; Anisimov, V. I. Spin State Transition and Covalent Bonding in $\mathrm{LaCoO}_{3}$. Phys. Rev. B 2012, 86, 195104.

(38) Smirnova, I. S. Normal Modes of the $\mathrm{LaMnO}_{3}$ Pnma Phase: Comparison with $\mathrm{La}_{2} \mathrm{CuO}_{4}$ Cmca Phase. Phys. B Condens. Matter 1999, 262, 247-261.

(39) Yan, J.-Q.; Zhou, J.-S.; Goodenough, J. B. Bond-Length Fluctuations and the Spin-State Transition in $\mathrm{LCoO}_{3}$ ( L = La, Pr, and Nd). Phys. Rev. B 2004, 69, 134409. 


\section{TABLE OF CONTENTS:}

TOC Graphic

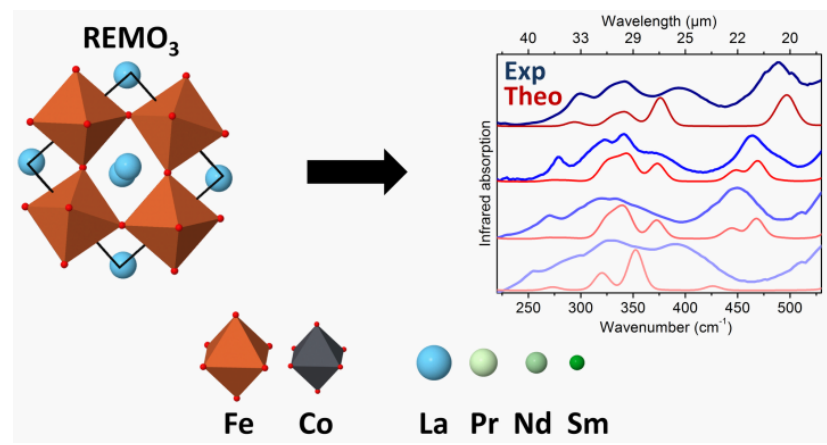

